\title{
On the restricted Waring problem over $\mathbb{F}_{2^{n}}[t]$
}

\author{
by \\ Luis Gallardo (Brest)
}

1. Introduction. The Waring problem for polynomial cubes over a finite field $F$ of characteristic 2 consists in finding the minimal integer $m \geq 0$ such that every sum of cubes in $F[t]$ is a sum of $m$ cubes. It is known that for $F$ distinct from $\mathbb{F}_{2}, \mathbb{F}_{4}, \mathbb{F}_{16}$, each polynomial in $F[t]$ is a sum of three cubes of polynomials (see [3]).

If a polynomial $P \in F[t]$ is a sum of $n$ cubes of polynomials in $F[t]$ such that each cube $A^{3}$ appearing in the decomposition has degree $<\operatorname{deg}(P)+3$, we say that $P$ is a restricted sum of $n$ cubes.

The restricted Waring problem for polynomial cubes consists in finding the minimal integer $m \geq 0$ such that each sum of cubes $S$ in $F[t]$ is a restricted sum of $m$ cubes.

The best known result for the above problem is that every polynomial in $\mathbb{F}_{2^{n}}[t]$ of sufficiently high degree that is a sum of cubes, is a restricted sum of eleven cubes. This result was obtained by the circle method in [1].

Here we improve this result using elementary methods. Let $F$ be a finite field of characteristic 2 , distinct from $\mathbb{F}_{2}, \mathbb{F}_{4}, \mathbb{F}_{16}$. In Theorem 7 , we prove that every polynomial in $F[t]$ is a restricted sum of at most nine cubes, and that every polynomial in $\mathbb{F}_{16}[t]$ is a restricted sum of at most ten cubes.

We also prove, in Theorem 9 , that by adding to a given $P \in \mathbb{F}_{2^{n}}[t]$ some square $B^{2}$ with $\operatorname{deg}\left(B^{2}\right)<\operatorname{deg}(P)+2$, the resulting polynomial is a restricted sum of at most four cubes, for all $n \neq 2$.

2. Sums of cubes. We consider a polynomial $P \in F[t]$ with $F$ a field of characteristic 2. We want to write $P$ as a restricted sum of cubes. In Lemma 5 we approach $P$ by a sum of two cubes $A^{3}+B^{3}$. This requires that $F$ be distinct from $\mathbb{F}_{4}$. Applying two more times the same reduction we are reduced to writing a polynomial of degree $<\operatorname{deg}(P) / 3+1$ as a sum of cubes. Specializing $F$ to a finite field distinct from $\mathbb{F}_{2}, \mathbb{F}_{4}, \mathbb{F}_{16}$, we obtain Theorem 6 ,

2000 Mathematics Subject Classification: Primary $11 T 55$. 
using the Serre Identity (see Lemma 2). For $F=\mathbb{F}_{16}$ a specific identity is used. The reduction requires that $P$ has degree higher than some constant integer $n$. We finish the reduction in Theorem 7, proving in a case by case manner the result for all polynomials of degree less than this constant $n$.

Lemma 1. Let $F$ be a finite field of characteristic $2, F \neq \mathbb{F}_{4}$ and $g \in$ $F, g \neq 0$. There exist $a, b \in F, a \neq 0$, such that $g=a^{3}+b^{3}$.

Proof. See [2].

Lemma 2 (Serre Identity). Let $F$ be a finite field of characteristic 2, distinct from $\mathbb{F}_{2}, \mathbb{F}_{4}, \mathbb{F}_{16}$. Every polynomial $P \in F[t]$ is a sum of three cubes, say $P=A^{3}+B^{3}+C^{3}$, with $A, B, C \in F[t], \operatorname{deg}(A)=\operatorname{deg}(B)=\operatorname{deg}(C)=$ $\operatorname{deg}(P)$.

Proof. This follows from the Serre formula

$$
b^{6}+a^{6}+a b c^{3} t=\left(a t+b^{2}\right)^{3}+\left(b t+a^{2}\right)^{3}+(c t)^{3}
$$

where $a, b, c$ are nonzero elements in $F$ such that $a^{3}+b^{3}+c^{3}=0$. See [3].

COROLlary 3. Let $F$ be a finite field of characteristic 2, distinct from $\mathbb{F}_{2}, \mathbb{F}_{4}, \mathbb{F}_{16}$. There exist three linear polynomials $A, B, C \in F[t]$ such that $t^{2}=A^{3}+B^{3}+C^{3}$.

Proof. By a specialization of variables in formula (1) we obtain $t=$ $U^{3}+V^{3}+W^{3}$, where $U, V, W \in F[t]$ and $\operatorname{deg}(U)=\operatorname{deg}(V)=\operatorname{deg}(W)=1$. Replace $t$ by $1 / t$ in this last formula, and then multiply both sides by $t^{3}$.

Lemma 4. Let $F \neq \mathbb{F}_{4}$ be a field of characteristic 2 . Let $n \geq 1$ be an integer, and $P \in F[t]$ a polynomial with $\operatorname{deg}(P) \in\{3 n+3,3 n+2,3 n+1\}$. There exist polynomials $A, B, Q \in F[t]$ such that $P=A^{3}+B^{3}+Q$. Moreover $\operatorname{deg}(A)=n+1, \operatorname{deg}(B) \leq n+1, \operatorname{deg}(Q) \leq 2 n+1$.

Proof. Set $P=\sum_{j=0}^{3 n+3} p_{j} t^{j}, d=\operatorname{deg}(P), S=\sum_{j=0}^{n} s_{j} t^{j}, A=a t^{n+1}+S$, $B=\alpha t^{n+1}+\beta t^{n}+\gamma t^{n-1}$, where the $\left\{s_{j}\right\}_{j=0, \ldots, n}$, and $a, \alpha, \beta, \gamma \in F$ are to be determined. If $d=3 n+3$, then we set $\beta=0, \gamma=0$. If $d=3 n+2$, then we set $s_{n}=0, a=1, \alpha=1, \gamma=0$. If $d=3 n+1$, then we set $s_{n}=0, s_{n-1}=0, a=1, \alpha=1, \beta=0$. Set $Q=P+A^{3}+B^{3}$. For $j$ from $2 n+2$ to $3 n+3$, we force all coefficients $q_{j}$ of $Q$ to be 0 , as follows. From the equations $q_{3 n+3}=a^{3}+\alpha^{3}+p_{3 n+3}=0, q_{3 n+2}=a^{2} s_{n}+\beta \alpha^{2}+$ $p_{3 n+2}=0, q_{3 n+1}=a^{2} s_{n-1}+\beta^{2} \alpha+a s_{n}^{2}+\alpha^{2} \gamma+p_{3 n+1}=0$, we obtain the missing values of $\alpha, a, \beta, \gamma, s_{n}, s_{n-1}$. More precisely, if $d=3 n+3$, then we get $a \neq 0$ from Lemma $1, \alpha$ from the equation $q_{3 n+3}=0, s_{n}$ from the equation $q_{3 n+2}=0$, and $s_{n-1}$ from the equation $q_{3 n+1}=0$; if $d=3 n+2$, then we get $\beta$ from the equation $q_{3 n+2}=0$, and $s_{n-1}$ from the equation $q_{3 n+1}=0$; if $d=3 n+1$, then we get $\gamma$ from the equation $q_{3 n+1}=0$. So the proof is finished for $n=1$, and we now take $n \geq 2$. Given an integer $k$ such 
that $1 \leq k \leq n-1$, suppose that we have determined $s_{n}$ to $s_{n-k}$ from the equations $q_{3 n+3}=0$ to $q_{3 n-k+2}=0$. We can then determine $s_{n-k-1}$ from the equation $q_{3 n-k+1}=0=a^{2} s_{n-k-1}+p_{3 n-k+1}+R$, where $R$ is a cubic form in $a, \alpha, \beta, \gamma$, and the $\left\{s_{j}\right\}_{n-k \leq j \leq n}$.

We now show the result of our reduction applied to a polynomial $P \in$ $F[t]$, where $F$ is a finite field of characteristic 2 , distinct from $\mathbb{F}_{4}$ :

Lemma 5. Let $F$ be a finite field of characteristic $2, F \neq \mathbb{F}_{4}$, and let $P \in$ $F[t]$ be a polynomial of degree $d \geq 4$. There exist polynomials $A, B, Q \in F[t]$ such that $P=A^{3}+B^{3}+Q$. Moreover $\operatorname{deg}\left(A^{3}\right)<d+3, \operatorname{deg}\left(B^{3}\right)<d+3$, $\operatorname{deg}\left(Q^{3}\right) \leq 2 d+e$, where $e=-3$ if $d \equiv 0 \bmod 3$; $e=-1$ if $d \equiv 2 \bmod 3$; $e=1$ if $d \equiv 1 \bmod 3$.

Proof. This follows from Lemma 4.

TheOREM 6. Let $F$ be a finite field of characteristic 2, distinct from $\mathbb{F}_{2}, \mathbb{F}_{4}, \mathbb{F}_{16}$. Every polynomial $P \in F[t]$ with $\operatorname{deg}(P)>6$ is a restricted sum of at most nine cubes. Every polynomial $P \in \mathbb{F}_{16}[t]$ with $\operatorname{deg}(P)>6$ is a restricted sum of at most ten cubes.

Proof. Suppose $F \neq \mathbb{F}_{2}, \mathbb{F}_{4}, \mathbb{F}_{16}$. If $\operatorname{deg}(P)>9$, we apply Lemma 5 three times and Lemma 2 once. If $7 \leq \operatorname{deg}(P) \leq 9$, we apply Lemma 5 twice and Lemma 2 once. For $F=\mathbb{F}_{16}$ the proof is the same, upon replacing the Serre formula in Lemma 2 by the identity

$$
t=(t r+s)^{3}+(t r+s+1)^{3}+\left(t+s r^{2}\right)^{3}+\left(t+(1+s) r^{2}\right)^{3},
$$

where $r \in \mathbb{F}_{16}$ satisfies $r^{4}=r+1$, and $s=r^{5}$.

TheOREM 7. Let $F$ be a finite field of characteristic 2, distinct from $\mathbb{F}_{2}, \mathbb{F}_{4}, \mathbb{F}_{16}$. Every polynomial $P \in F[t]$ is a restricted sum of at most nine cubes. Every polynomial $P \in \mathbb{F}_{16}[t]$ is a restricted sum of at most ten cubes.

Proof. From Theorem 6, we can assume that $\operatorname{deg}(P) \leq 6$. Suppose $F \neq \mathbb{F}_{16}$. If $\operatorname{deg}(P) \leq 1$ the result follows from the Serre identity in Lemma 2 . Suppose $\operatorname{deg}(P)=2$ and write $P=a_{2} t^{2}+a_{1} t+a_{0}$. From Corollary $3, a_{2} t^{2}=$ $\left(a_{2}^{1 / 2} t\right)^{2}$ is a sum of 3 cubes of polynomials of degree 1 , but $\operatorname{deg}\left(P+a_{2} t^{2}\right) \leq 1$, so $P=\left(P+a_{2} t^{2}\right)+a_{2} t^{2}$ is a sum of at most 6 cubes, each of degree $\leq 1$. Suppose $\operatorname{deg}(P)=3$ and write $P=a_{3} t^{3}+P_{2}$ with $\operatorname{deg}\left(P_{2}\right) \leq 2$. By Lemma 1 , $a_{3}=a^{3}+b^{3}$ with some $a, b \in F$; so $a_{3} t^{3}=(a t)^{3}+(b t)^{3}$; it follows that $P$ is a sum of at most 8 cubes, each of degree $\leq 1$. Suppose $\operatorname{deg}(P)=4$ and write $P=t^{3} P_{1}+P_{2}$ with $\operatorname{deg}\left(P_{1}\right)=1$ and $\operatorname{deg}\left(P_{2}\right)=2$. Apply Lemma 2 to $P_{1}$ and $P_{2}$. We deduce that $P$ is a sum of at most 6 cubes, each of degree $\leq 2$. Suppose $\operatorname{deg}(P)=5$. By Lemma $4, P=A^{3}+B^{3}+P_{3}$ with $\operatorname{deg}(A) \leq 2, \operatorname{deg}(B) \leq$ 2 and $\operatorname{deg}\left(P_{3}\right) \leq 3$. By Lemma $1, P_{3}=(c t)^{3}+(d t)^{3}+P_{2}$ with some $c, d \in F$ and $\operatorname{deg}\left(P_{2}\right) \leq 2$; so that by Lemma $2, P_{2}$ is a sum of at most 3 cubes, each of degree $\leq 2$. Hence $P$ is a sum of at most 7 cubes, each of degree $\leq 2$. Suppose 
$\operatorname{deg}(P)=6$. By Lemma $4, P=A^{3}+B^{3}+P_{4}$ with $\operatorname{deg}(A) \leq 2, \operatorname{deg}(B) \leq 2$ and $\operatorname{deg}\left(P_{4}\right) \leq 4$. So $P$ is a sum of at most 8 cubes, each of degree $\leq 2$. The proof is similar when $F=\mathbb{F}_{16}$, with the appeal to Lemma 2 replaced by the identity (2), and Corollary 3 replaced by a similar result obtained after replacing $t$ by $1 / t$ and multiplying both sides of (2) by $t^{3}$.

3. Allowing a square. We consider a polynomial $P \in F[t]$, where $F$ is a perfect field of characteristic 2 . We approach the square root $S$ of the derivative of $P$ relative to $t$ by a sum of at most two polynomials, say $U, V$, of the form $A^{2} B+t B^{3}$. The reduced polynomial $Q=S+U+V$ is of degree close to $\operatorname{deg}(S) / 3$ (see Lemma 8 ). This reduction requires that every element in $F$ is a sum of at most two cubes. So we specialize $F$ to a finite field other than $\mathbb{F}_{4}$, and we apply the identity $T=(T+1)^{3}+T^{3}+(T+1)^{2}$ to the polynomial $t W^{2}$. The main result is Theorem 9 .

LEMma 8. Let $F$ be a perfect field of characteristic 2 such that every element in $F$ is a sum of at most two cubes. Let $n \geq 0$ be an integer, and $S \in F[t]$ be a polynomial with $\operatorname{deg}(S) \in\{3 n+2,3 n+1,3 n\}$. There exist polynomials $A, B, C, D, Q \in F[t]$ such that

$$
S=A^{2} B+t B^{3}+C^{2} D+t D^{3}+Q,
$$

where $\operatorname{deg}(B)=n, \operatorname{deg}(C) \leq n, \operatorname{deg}(D) \leq n, \operatorname{deg}(Q)<n-1$. Moreover, if $\operatorname{deg}(S) \in\{3 n, 3 n+1\}$ then $\operatorname{deg}(A) \leq n$; if $\operatorname{deg}(S)=3 n+2$ then $\operatorname{deg}(A)=$ $n+1$.

Proof. Suppose that $n \geq 1$. Set $S=\sum_{j=0}^{3 n+3} p_{3 n+3-j} t^{3 n+3-j}, A=$ $a t^{n+1}+\sum_{k=0}^{n} a_{k} t^{k}, B=c t^{n}, C=\sum_{k=0}^{n} c_{k} t^{k}, D=d t^{n}+t^{n-1}$. If $p_{3 n+1}=0$, then we set $c=d=1$. If $p_{3 n+1} \neq 0$, then by hypothesis we obtain $c, d \in F$, $c \neq 0$, such that $p_{3 n+1}=c^{3}+d^{3}$. If $p_{3 n+2}=0$, then we take $a=0$. If $p_{3 n+2} \neq$ 0 , then we take $c \neq 0$ from $c a^{2}=p_{3 n+2}$. We now determine the $\left\{c_{k}, a_{k}\right\}_{0 \leq k \leq n}$ such that all monomials $\left\{r_{s} t^{s}\right\}_{n \leq s \leq 3 n}$ of $S+A^{2} B+t B^{3}+C^{2} D+t D^{3}$ are 0 , as follows. From the linear equation $r_{3 n-1}=c_{n}^{2}+d+p_{3 n-1}=0$, we obtain $c_{n}$, then from the linear equation $r_{3 n}=c a_{n}^{2}+d^{2}+c_{n}^{2} d+p_{3 n}=0$, we obtain $a_{n}$. From the linear equation $r_{3 n-3}=c_{n-1}^{2}+p_{3 n-3}=0$, we obtain $c_{n-1}$, then from the linear equation $r_{3 n-2}=c a_{n-1}^{2}+1+c_{n-1}^{2} d+p_{3 n-2}=0$, we obtain $a_{n-1}$. This finishes the proof for $n=1$, and so we now take $n \geq 2$. From the linear equation $r_{3 n-5}=c_{n-2}^{2}+p_{3 n-5}=0$, we obtain $c_{n-2}$, then from the linear equation $r_{3 n-4}=c a_{n-2}^{2}+c_{n-2}^{2} d+p_{3 n-4}=0$, we obtain $a_{n-2}, \ldots$ Finally, we obtain $c_{0}$ from the linear equation $r_{n-1}=c_{0}^{2}+p_{n-1}=$ 0 , and $a_{0}$ from the linear equation $r_{n}=c a_{0}^{2}+d c_{0}^{2}=0$. So the resulting polynomial $Q=S+A^{2} B+t B^{3}+C^{2} D+t D^{3}$ is of degree less than or equal to $n-2$, finishing the proof. The proof for $n=0$ is similar by setting $A=a t+a_{0}, B=c, C=c_{0}, D=d$. 
TheOREM 9. Let $F$ be a finite field of characteristic 2 , distinct from $\mathbb{F}_{4}$, and let $P \in F[t]$. There exists a square $B^{2}$ in $F[t]$ with $\operatorname{deg}\left(B^{2}\right)<\operatorname{deg}(P)+2$ such that $P+B^{2}$ is a restricted sum of at most four cubes.

Proof. For any $H \in F[t]$ we write $H^{\prime}$ for the derivative of $H$ relative to $t$. Put $P^{\prime}=S^{2}$, and $d=\operatorname{deg}(S) \in\{3 n+2,3 n+1,3 n\}$ for some integer $n \geq 0$. Now $P=(t P)^{\prime}+t P^{\prime}$, where $(t P)^{\prime}$ is a square in $F[t]$ of degree $<\operatorname{deg}(P)+2$. So it suffices to prove the result for $t P^{\prime}$. Applying Lemmas 1 and 8 to $S$ we get

$$
\left(t P^{\prime}\right)^{\prime}=S^{2}=K^{2} K^{\prime}+L^{2} L^{\prime}+Q^{2}
$$

with $K=A^{2}+t B^{2}, L=C^{2}+t D^{2}$. Then $\operatorname{deg}(L) \leq 2 n+1$. Also $\operatorname{deg}(K)=$ $2 n+1$ if $d \equiv 0$ or $1 \bmod 3 ; \operatorname{deg}(K)=2 n+2$ if $d \equiv 2 \bmod 3$. Furthermore, $\operatorname{deg}(Q)<n-1$. Integrating (3) over $t$, we get

$$
R^{2}+t P^{\prime}=K^{3}+L^{3}+t Q^{2}
$$

for some $R \in F[t]$. We have $\operatorname{deg}\left(L^{3}\right) \leq 6 n+3<6 n+4 \leq \operatorname{deg}\left(t P^{\prime}\right)+3$. If $d \equiv 0 \bmod 3$ or $d \equiv 1 \bmod 3$ then $\operatorname{deg}\left(K^{3}\right) \leq 6 n+3<6 n+4 \leq \operatorname{deg}\left(t P^{\prime}\right)+3$. If $d \equiv 2 \bmod 3$ then $\operatorname{deg}\left(K^{3}\right)=6 n+6<6 n+8 \leq \operatorname{deg}\left(t P^{\prime}\right)+3$. Now $\operatorname{deg}\left(\left(t Q^{2}\right)^{2}\right) \leq \operatorname{deg}\left(\left(t Q^{2}\right)^{3}\right)<6 n-3<\operatorname{deg}\left(t P^{\prime}\right)+2<\operatorname{deg}\left(t P^{\prime}\right)+3$. If $d \equiv 0 \bmod 3$ or $d \equiv 1 \bmod 3$ then, using $R^{2}=t P^{\prime}+K^{3}+L^{3}+t Q^{2}$, we obtain $\operatorname{deg}\left(R^{2}\right) \leq 6 n+3$; i.e. $\operatorname{deg}\left(R^{2}\right)<6 n+3 \leq \operatorname{deg}\left(t P^{\prime}\right)+2$. Similarly, $\operatorname{deg}\left(R^{2}\right) \leq 6 n+6<6 n+7 \leq \operatorname{deg}\left(t P^{\prime}\right)+2$ when $d \equiv 2 \bmod 3$. From the identity $T=(T+1)^{3}+T^{3}+(T+1)^{2}$, we obtain

$$
t P^{\prime}=K^{3}+L^{3}+\left(t Q^{2}+1\right)^{3}+\left(t Q^{2}\right)^{3}+\left(R+t Q^{2}+1\right)^{2} .
$$

This establishes the result.

\section{References}

[1] M. Car et J. Cherly, Sommes de cubes dans l'anneau $\mathbb{F}_{2^{h}}[X]$, Acta Arith. 65 (1993), $227-241$.

[2] R. Lidl and H. Niederreiter, Finite Fields, Cambridge Univ. Press, 1984, pp. 327 and 295.

[3] L. N. Vaserstein, Sums of cubes in polynomial rings, Math. Comp. 56 (1991), 349357.

Department of Mathematics

University of Brest

6, Avenue le Gorgeu

29285, Brest Cedex, France

E-mail: Luis.Gallardo@univ-brest.fr

Received on 20.2.1998

and in revised form on 29.9.1999 